\title{
The effect of contrast on the completeness of binocular rivalry suppression
}

\author{
MARK HOLLINS \\ University of North Carolina, Chapel Hill, North Carolina 27514
}

\begin{abstract}
Binocular rivalry between orthogonal sine wave gratings was studied by asking subjects to indicate when they saw just one or the other grating, as opposed to a composite. Using this measure, it was found that the completeness of rivalry (1) usually increased with grating contrast; (2) increased as a trial progressed, up to about 40 sec; and (3) depended on spatial frequency, with the optimal spatial frequency being lower than that at which contrast sensitivity was maximal. These findings supplement what has been learned about rivalry by other methods.
\end{abstract}

The term binocular rivalry refers to the fluctuating perceptual experience that occurs when dissimilar targets are presented to the two eyes. The phenomenon is often thought to consist entirely of periods during which the two targets are alternately rendered invisible. A now standard procedure for tracking the course of rivalry was introduced by Breese (1899, 1909), who required his subjects to indicate when the left eye's target dominated and when the right eye's did so; no third alternative was provided. Widespread use of this method since Breese's time has probably helped to foster the view that periods in which both targets are visible either do not occur or are of no theoretical consequence.

Under some conditions, however, there are periods when an actual blend or superposition of the targets is perceived, with contours from the two eyes seen as intersecting (Ogle \& Wakefield, 1967). In addition, there can be periods when a mosaic composed of interspersed fragments of the two targets is seen (e.g., Hochberg, 1964). Panum (1858, p. 38), for example, describes the rivalry of a pair of orthogonal gratings as follows:

The resulting combined percept in the joint visual field cannot be drawn accurately because of its constant, restless change. Sometimes the diagonal lines of one target are seen, sometimes those of the other. Usually, however, some of both are present together, so that in one place the diagonal lines of one target predominate, in another place, those of the other target; while in still another place both are visible, but faintly, as if effaced by washing or wiping.

The question of when such composite perceptions occur is the subject of the present investigation. Because rivalry may, almost by definition, be called more vigorous when targets alternate crisply and in

This research was supported by NSF Grant BNS 77-23050. The technical contributions of George Bailey are gratefully acknowledged. their entirety than when they blend together into a composite, the question is an important one for identifying the factors that promote rivalry, and for gaining some insight into the physiological mechanisms that underlie it.

The procedure used in the present study is one employed by Wade $(1974,1975)$, who asked subjects to indicate when they perceived only the left eye's target, when they perceived only the right eye's, and when they perceived a composite of the two views. This procedure has been called the method of exclusive visibility (Hollins \& Leung, 1978), because it requires subjects to indicate when a given target is visible to the exclusion of the other.

In the data to be reported here, no distinction is made between the two possible types of composites: mosaics consisting of interspersed portions of the two targets, and blends in which intersecting contours can be seen. It would have been difficult to record such distinctions, since, in accordance with Panum's observations, the two types of composites were often seen simultaneously in different regions of the target area.

In the present study, the method of exclusive visibility is used to measure the completeness of binocular rivalry for different levels of grating contrast. If the amount of exclusive visibility were found to increase with contrast, this would help to explain the fact that perceptual blending is seldom reported by investigators using high-contrast targets.

Another question addressed in the present series of experiments is whether the completeness of rivalry changes systematically over the course of a viewing period. Finally, a determination is made of the spatial frequencies that are most effective in producing exclusive visibility.

\section{METHODS}

Stimuli

The targets were sine wave gratings produced by standard methods (Campbell \& Green, 1965) on a Tektronix 5110 oscil- 
loscope with a rapidly decaying (P31) phosphor and no graticle Voltage signals were provided to it by Wavetek Model 171 function generators. A 1-MHz triangular-wave input to the vertical amplifier produced a raster, refreshed 2,000 times per second, which appeared as a steady, uniform field with a luminance of $1.4 \mathrm{~cd} / \mathrm{m}^{2}$. Sinusoidal input from a second function generator modulated the intensity of the beam to produce the gratings, without changing the space-averaged luminance of the screen. For stationary gratings, the same function generator that modulated beam intensity was used to trigger the oscilloscope. For drifting gratings, an additional function generator at a slightly different frequency provided the trigger signal.

Using a chinrest, subjects looked into a box $102 \mathrm{~cm}$ in length to see the target, which was visible through a 1-deg square window, made of 2-mm-thick Plexiglas, in its rear wall. The inner surfaces of the box were painted flat black. Within the apertures for the subject's eyes in the front of the box were rotary prisms, to allow the image of the target to be shifted horizontally in either eye to facilitate fusion. Inside the box, just behind each aperture, was a Dove prism. The one for the right eye rotated the image of the grating so that it appeared horizontal, while the one for the left eye was oriented so that this eye saw the grating as vertical. The left eye's Dove prism was used so that any prism-dependent optical changes, such as a reduction in contrast, would be equivalent in the two images.

Bars of light, to aid in fusion, were adjacent to the target on all four sides. The bars above and below the target were oriented horizontally; those on the sides were oriented vertically. These bars were $1 \mathrm{deg}$ in length and $9 \mathrm{~min}$ wide, and were separated from the target square by a dark zone $14 \mathrm{~min}$ of arc wide. Frosted glass attached to the outside of the box covered the windows that defined these bars, so that light from the oscilloscope, which transilluminated them, was diffused, giving them a uniform luminance some $.14 \log$ unit lower than the space-averaged luminance of the target square.

No fixation point was used; subjects were instructed to look approximately in the center of the target, but to shift their gaze slightly from time to time to prevent Troxler fading, which can occur even at the fovea under conditions of strict fixation (Crovitz \& Lockhead, 1967). This maneuver was apparently successful, for subjects reported that seldom, if ever, did both gratings disappear at the same time.

\section{Calibrations}

Luminance was measured with an S.E.I. photometer from the subject's point of view, with the prisms in place.

Grating contrast was adjusted with a logarithmic potentiometer attached to a large dial. The potentiometer was calibrated by measuring its output voltage on a storage oscilloscope. For a particular dial setting, this peak-to-peak voltage was the same for all signal frequencies used in this study,

Absolute grating contrast, defined as $\left(\mathrm{L}_{\max }-\mathrm{L}_{\min }\right) /\left(\mathrm{L}_{\max }+\mathrm{L}_{\min }\right)$, was measured by covering the target oscilloscope screen with black cardboard containing a narrow vertical slit. Light passing through the slit fell onto the photodiode of a United Detector Technology IIA radiometer, and the output of the diode was measured on the storage oscilloscope. During these measurements, a low spatial frequency of grating was used, and was made to drift slowly across the screen. Measurements using this technique could not be made at high spatial frequencies. It is assumed in this paper that the contrast produced by a certain voltage is independent of spatial frequency; this probably errs in the direction of assigning too high a contrast to the finer gratings. It was found that contrast increased linearly with voltage until a contrast of about .65 was reached; therefore, only values below that level were used in the experiments.

\section{Subjects}

Three men and one woman (M.S.) participated in this study. M.H. (the author) was 32, K.H. 29, M.S. 21 , and K.E. 20 years of age at the time of the experiments. None had a history of ab- normal binocular vision, and all could appreciate random dot stereograms. While M.H., K.H., and K.E. experienced no difficulty in maintaining fusion of the stimulus array with the rotary prisms set at zero, M.S. required a setting of 3 prism diopters base in for each eye.

\section{Recording of Responses}

Subjects M.H. and K.H. signaled fluctuations of rivalry by operating a three-way toggle switch, pushing it one way when only the vertical grating was seen and the other way when only the horizontal grating was seen. When a composite of the two was perceived, the switch was allowed to return to the center position. The amount of exclusive visibility within a trial was cumulated separately on Hunter Klockounters for the left and right eyes. For subjects M.S. and K.E., a different switch was used, consisting of a lever pivoted in the center. Subjects depressed one end or the other of the lever to indicate exclusive visibility, thus closing one of a pair of microswitches. Both microswitches were open when the lever was released. For these two subjects, the time of occurrence and duration of every response were recorod on a PDP $11 / 03$ computer.

\section{Procedure}

At the beginning of each run, the subject adapted for $5 \mathrm{~min}$ to the uniformly illuminated target square. At the end of this period, the uniform field was replaced by the grating, and the subject recorded exclusive visibility for a 100 -sec trial. Successive trials were separated from one another by 100 -sec rest periods, during which the subject viewed the uniform square. Because it is known that prolonged periods of binocular rivalry can lead to reduced. amounts of exclusive visibility (Hudnell \& Hollins, 1979), runs were limited to seven or eight trials. Usually, only a single run per day was carried out on a given subject.

All measurements on M.S. and K.E. were made at a spatial frequency of 3 cycles/deg. Seven levels of contrast were presented in random order, five times each. As in the other experiments of this study, randomization was subject to the constraint that no target could be presented a second time until each had been presented once, and so on.

For M.H. and K.H., contrast and spatial frequency were both varied. Five levels of contrast were employed at 3, 6, and 9 cycles/ deg, but the lowest contrast was not used at more extreme spatial frequencies ( 1.54 and 12 cycles/deg) because of its proximity to contrast threshold. The 23 different stimulus conditions were presented in random order until eight trials had been run with each

For the determinations of contrast threshold with stationary gratings, one eye was patched while the other viewed the stimulus. After a 5-min period of adaptation to the uniform square, the grating was presented for $500 \mathrm{msec}$ once every $2 \mathrm{sec}$, and the subject adjusted the potentiometer dial which controlled the contrast until the grating could just be seen. The experimenter recorded this value and spun the dial to some different position in prepara tion for the next trial. Successive blocks of 10 trials were presented to one eye or the other according to an ABBA design, $5 \mathrm{~min}$ of light adaptation to the homogeneous raster being employed whenever there was a change of eye. Spatial frequency was randomized within a block of trials. In all, eight determinations were made at each spatial frequency for each eye.

To measure the contrast threshold for the detection of movement, gratings drifting at $5 \mathrm{~Hz}$ were presented continuously during a trial, but were replaced by the homogeneous raster once the subject had made a setting. Otherwise, the protocol was similar to that followed in determining thresholds for stationary gratings.

\section{RESUL.TS}

The course of rivalry for an individual trial is illustrated in Figure 1. These data are for subject K.E., viewing 3-cycle/deg gratings with a contrast of .42 . 


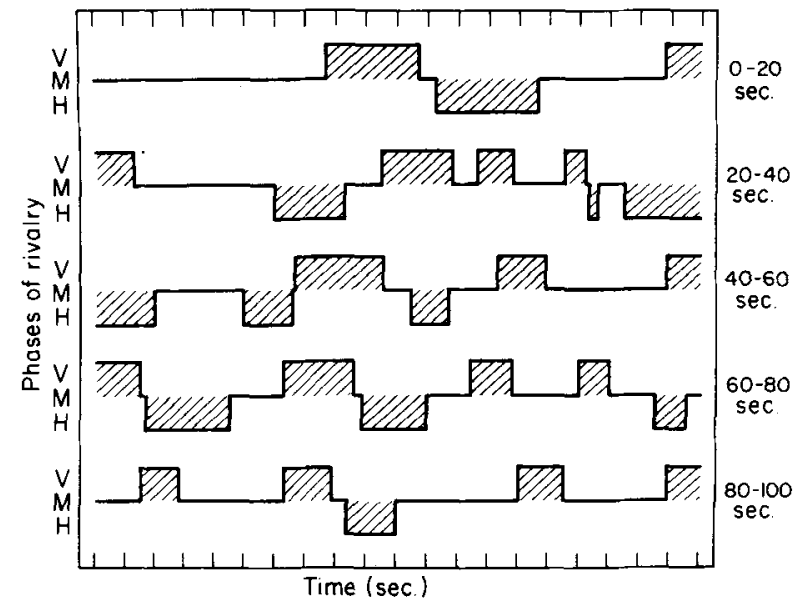

Figure 1. The course of binocular rivalry during a 100-sec trial. Ticks on the abscissa mark off 1-sec intervals. The top trace corresponds to the first 20 sec of the trial, the second trace to the next 20 sec, and so on. When the trace is elevated, only the vertical grating (V) was perceived; when the trace is depressed, only the horizontal grating (H) was perceived. Hatched areas thus represent periods of exclusive visibility. This figure was drawn on the basis of data tabulated on Subject K.E., using 3-cycle/deg gratings with a contrast of $\mathbf{4 2}$.

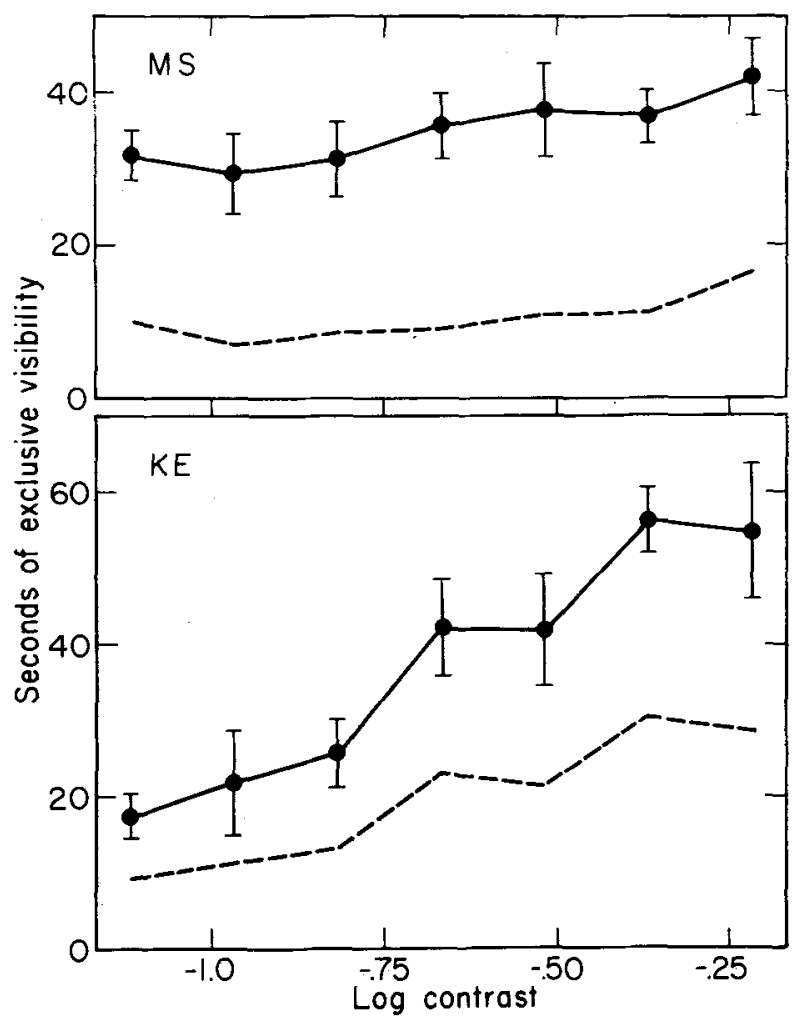

Figure 2. Total number of seconds of exclusive visibility occurring during a trial, as a function of grating contrast. Error bars indicate one standard error above and another below each point, which is the mean result of five trials. Dashed line indicates the amounts of exclusive visibility cumulated by the left eye only. Spatial frequency was 3 cycles/deg.
The top line in the figure corresponds to the first $20 \mathrm{sec}$ of the trial, the second line to the next $20 \mathrm{sec}$, and so on; hatched areas indicate periods of exclusive visibility. Upward deflections of the line indicate periods in which only the vertical grating was seen; downward deflections indicate periods in which only the horizontal grating was seen. Successive phases of exclusive visibility, which can belong either to the same or to different eyes, are usually separated by periods in which a composite of the two gratings is perceived. There is a suggestion in the figure that the amount of exclusive visibility increases as the trial proceeds, a subject on which more is said below.

Amounts of exclusive visibility obtained with different levels of contrast are shown in Figure 2. Each point is the mean result of five trials, with the error bars indicating one standard error above and below the mean. It can be seen that the amount of exclusive visibility increases with the contrast of the targets. The increase is small enough to have occurred by chance in the case of M.S., but is large and significant for K.E. (All statements of statistical significance in this paper are based on a Friedman two-way analysis of variance by ranks, with $p=.05$.) The dashed lines indicate the amount of exclusive visibility for the left eye. These lines tend upward as well, but more gradually than the solid lines, thus showing that each eye's exclusive visibility increases with contrast.

Table 1 breaks down these measurements of exclusive visibility into two factors: the mean duration of individual phases of rivalry and the mean number of occurrences of these phases during a trial. It can be seen that the rivalry speeds up as contrast is raised, in that more phases of every type occur. This is made

Table 1

Mean Duration (D) in Seconds and Mean Occurrences per Trial (O/T) of Phases of Rivalry

\begin{tabular}{|c|c|c|c|c|c|c|}
\hline \multirow{3}{*}{$\begin{array}{c}\text { Log } \\
\text { Contrast }\end{array}$} & \multicolumn{4}{|c|}{ Exclusive Visibility } & & \\
\hline & \multicolumn{2}{|c|}{ Vertical } & \multicolumn{2}{|c|}{ Horizontal } & \multicolumn{2}{|c|}{ Mixed } \\
\hline & D & $\mathrm{O} / \mathrm{T}$ & D & $\mathrm{O} / \mathrm{T}$ & D & $\mathrm{O} / \mathrm{T}$ \\
\hline \multicolumn{7}{|c|}{ Subject M.S. } \\
\hline $\begin{array}{l}-1.12 \\
-.97 \\
-.82 \\
-.67 \\
-.52 \\
-.37 \\
-.22\end{array}$ & $\begin{array}{l}.62 \\
.46 \\
.47 \\
.46 \\
.60 \\
.48 \\
.66\end{array}$ & $\begin{array}{l}16.2 \\
15.2 \\
18.2 \\
19.4 \\
18.2 \\
23.2 \\
24.8\end{array}$ & $\begin{array}{l}.91 \\
.86 \\
.78 \\
.85 \\
.93 \\
.83 \\
.83\end{array}$ & $\begin{array}{l}23.8 \\
26.0 \\
29.2 \\
31.2 \\
28.8 \\
31.0 \\
30.6\end{array}$ & $\begin{array}{l}1.67 \\
1.69 \\
1.43 \\
1.25 \\
1.30 \\
1.15 \\
1.04\end{array}$ & $\begin{array}{l}41.0 \\
41.8 \\
48.2 \\
51.4 \\
47.8 \\
55.0 \\
56.0\end{array}$ \\
\hline \multicolumn{7}{|c|}{ Subject K.E. } \\
\hline $\begin{array}{l}-1.12 \\
-.97 \\
-.82 \\
-.67 \\
-.52 \\
-.37 \\
-.22\end{array}$ & $\begin{array}{l}1.39 \\
1.55 \\
1.32 \\
2.23 \\
1.96 \\
2.00 \\
1.93\end{array}$ & $\begin{array}{r}6.6 \\
7.2 \\
10.0 \\
10.2 \\
10.8 \\
15.2 \\
14.8\end{array}$ & $\begin{array}{l}1.13 \\
1.43 \\
1.23 \\
1.86 \\
1.81 \\
1.99 \\
1.79\end{array}$ & $\begin{array}{r}7.2 \\
7.4 \\
10.0 \\
10.4 \\
11.4 \\
13.0 \\
14.6\end{array}$ & $\begin{array}{l}5.74 \\
5.15 \\
3.55 \\
2.76 \\
2.58 \\
1.57 \\
1.55\end{array}$ & $\begin{array}{l}14.4 \\
15.2 \\
21.0 \\
21.0 \\
22.6 \\
27.8 \\
29.2\end{array}$ \\
\hline
\end{tabular}




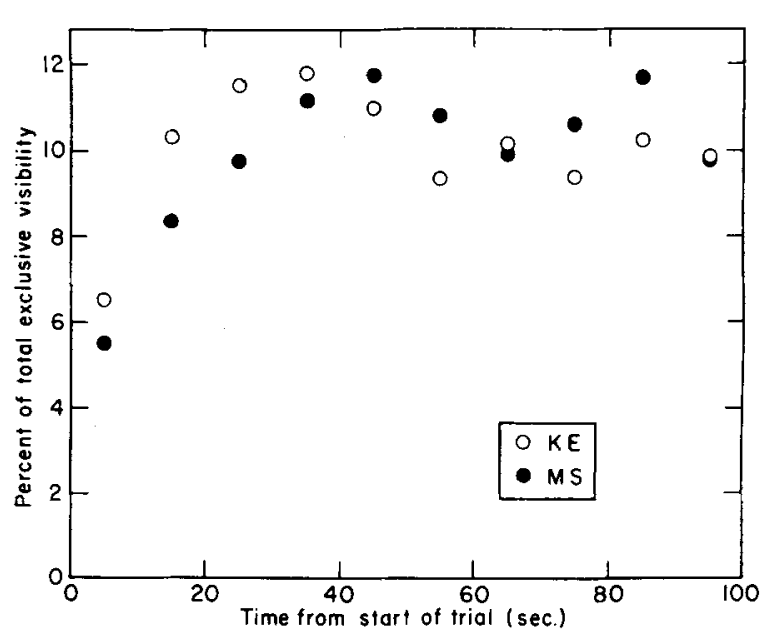

Figure 3. Percent of a trial's total exclusive visibility which occurred during each of its 10 -sec components. Pereentages were calculated separately for each level of contrast, then averaged across contrasts. Each point is plotted at the midpoint of the interval that it represents. Filled and unfilled circles show the data for Subjects M.S. and K.E., respectively.

possible primarily by a decrease in the length of the individual "mixed" phases, during which portions of both gratings are visible. These trends are large enough to attain significance for K.E., but not for M.S.

The subjects reported a clear impression that rivalry grew more vigorous as a trial proceeded. This possibility was investigated by determining the fraction of total exclusive visibility that occurred in each 10 -sec block of the data. The results of this analysis, averaged across levels of contrast with each given equal weight, are shown in Figure 3. The data from the two subjects are in close agreement in showing that exclusive visibility increases steadily during the first $40 \mathrm{sec}$ of a trial, and then levels off. Inspection of individual records showed that this result was associated sometimes with an increase in the number, and sometimes with an increase in the mean duration, of periods of exclusive visibility.

Subjects M.H. and K.H. tracked rivalry for five different spatial frequencies of grating. Their results are shown in Figure 4, where each point is the mean exclusive visibility for eight trials. Again, there is a tendency for exclusive visibility to increase with contrast, although this is much greater for one subject than for the other. The dependence of exclusive visibility on contrast was significant at every spatial frequency for K.H., but only at the three highest spatial frequencies for M.H.

It was found that by sliding the curves of Figure 4 horizontally to achieve maximum overlap, the data for the different spatial frequencies could be made to lie along a single continuous curve for each subject. The results of this shifting along the log contrast axis are shown in Figure 5. At low contrasts, exclusive visibility rises approximately linearly with $\log$ contrast, but, in the case of M.H., it levels off at high contrasts. Inspection of the data for this subject suggests that exclusive visibility does not vary significantly with contrast at 1.54 and 3 cycles/deg because these data are nearly confined to the high-contrast plateau defined by the data for the other spatial frequencies.

The amount of sliding necessary to bring the data for the different spatial frequencies into superimposition can be used as an indicator of the capacity of these different spatial frequencies to produce binocular rivalry. The spatial frequency "action spectrum" of rivalry determined in this way is shown by the filled circles in Figure 6. For each subject, 3 cycles/deg was the most effective spatial frequency, meaning that less contrast was required here than at other spatial frequencies to produce a particular amount of exclusive visibility.

This result is surprising when compared with the fact that contrast sensitivity, shown by the squares, was found to peak at 6 cycles/deg. These values are the average of the measurements made separately in each eye. It is unclear why low spatial frequency gratings should promote more vigorous rivalry than high spatial frequency gratings with an equal elevation above contrast threshold. Could the transient system, known to favor low spatial frequencies

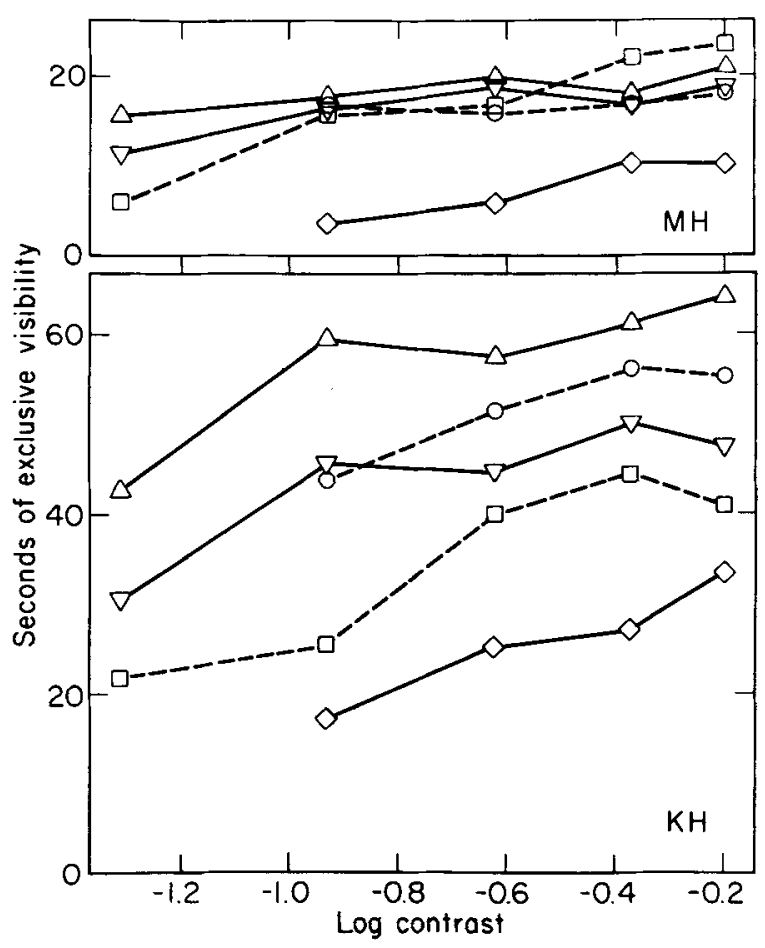

Figure 4. Number of seconds of exclusive visibility as a function of grating contrast for Subjects M.H. and K.H. Different symbols plot the results for different spatial frequencies: 1.54 cycles/deg $(O), 3$ cycles $/ \mathrm{deg}(\Delta), 6$ cycles/deg $(\nabla), 9$ cycles $/ \mathrm{deg}(\square), 12$ cycles/ deg $(\diamond)$. Each point is the mean result of eight trials. 


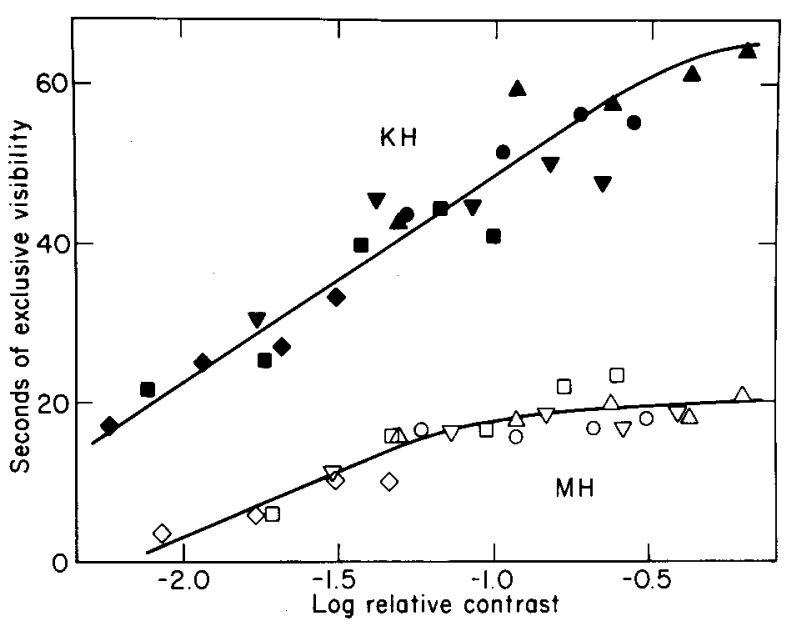

Figure 5. Replot of the data in Figure 4, with measurements for the different spatial frequencies shifted horizontally for maximum overlap. A single smooth curve has been drawn by eye through the data for each subject.

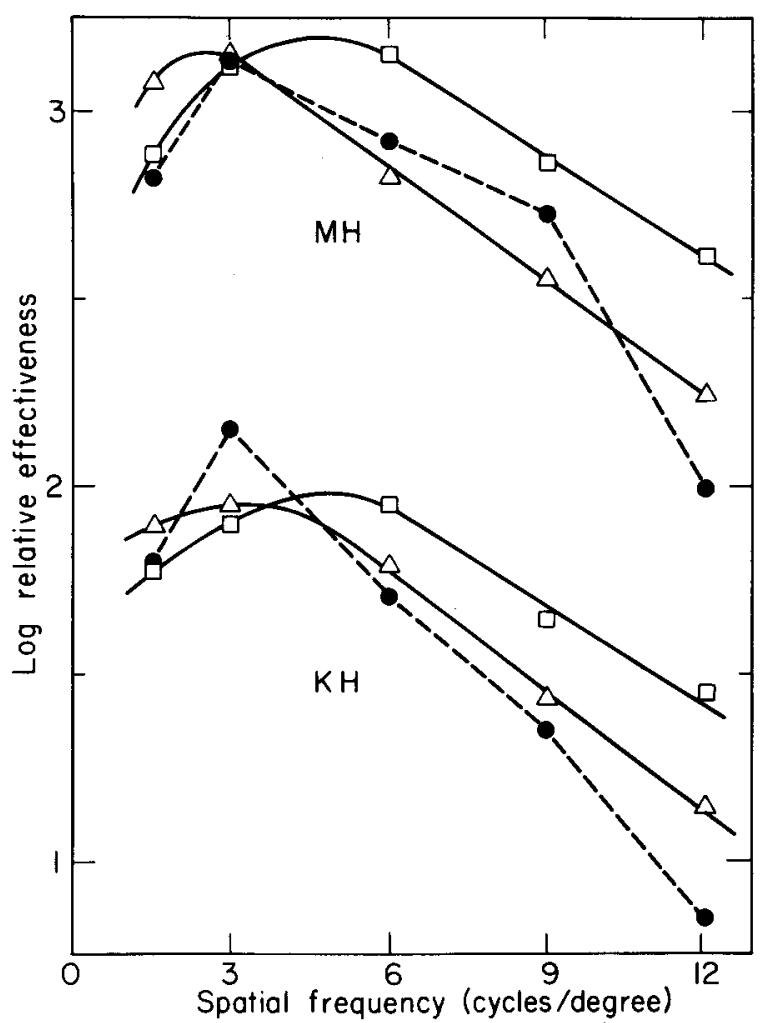

Figure 6. Spatial frequency action spectrum of binocular rivalry $(\bullet)$, based on the amount of shifting along the log contrast axis necessary to bring the data for different spatial frequencies into maximum overlap as illustrated in the previous figure. The more a set of data had to be shifted to the left, the less effective was that spatial frequency in promoting binocular rivalry. Unfilled symbols plot, for comparison, the contrast threshold for stationary gratings (squares) and for the perception of motion of drifting gratings (triangles). The relative positions of the various curves along the ordinate are arbitrary.
(Kulikowski \& Tolhurst, 1973), be involved in rivalry even of stationary targets, as in the present situation? To explore this notion, contrast sensitivity to movement was measured using drifting gratings, for comparison with the rivalry data. Subjects adjusted the contrast of the gratings until motion could just be observed, whether or not the individual stripes of the grating could be resolved. The results, averaged for the two eyes, are shown by the triangles in Figure 6 . This function, like the spatial frequency action spectrum of rivalry, peaks at 3 cycles/deg.

\section{DISCUSSION}

The major finding of this study is that the amount of exclusive visibility increases with the contrast of rivaling targets. High-contrast targets alternate more crisply than low-contrast targets, which frequently blend together into a composite. There is considerable individual variation, however: of four subjects, two showed a strong effect, one a negligible effect, and one an effect only under some conditions.

Whether measures of exclusive visibility correspond in any but an obvious phenomenal sense to the vigorousness of rivalry is not presently known. It might be asked, for example, whether rivalry suppression is deeper at a particular point within a target when the entire target is invisible than when only a small region centered on the point in question is invisible.

The only presently accepted method for measuring the depth of rivalry suppression is to superimpose a test flash on a rivaling target: threshold is higher when the background target is in the suppressed state than when it is dominant, with the amount of threshold elevation serving as a measure of suppression (Wales \& Fox, 1970). With this technique, the depth of suppression has been shown to be immune to various changes in the experimental conditions (Blake \& Camisa, 1979; Blake \& Lema, 1978; Fox \& Check, 1972).

The test flash in these studies has been presented during periods of complete dominance or suppression; measurements have not been made during mixed states. It will be recalled, however, that when rivaling contours are seen as intersecting, they often appear faded (Ogle \& Wakefield, 1967; Panum, 1858), suggesting that weak, or partial, suppression can occur during the mixed state. If this interpretation is correct, then the combined duration of the periods of exclusive visibility occurring during a viewing period could serve as an index of the overall strength of rivalry for that trial.

\section{Changes in Rivalry Strength During a Trial}

The second finding of the present study is that binocular rivalry grows progressively more vigorous during the first $\mathbf{4 0} \mathrm{sec}$ or so of a trial, beyond which 
point it levels off. Because the apparent contrast of gratings decreases during prolonged viewing (Blakemore, Muncey, \& Ridley, 1971, 1973), and because the completeness of rivalry depends on contrast, it might have been predicted that there would be less and less exclusive visibility as a trial progressed. The present result is thus surprising.

The mixed state is not a stable one: one moment the left grating will be slightly more dominant than the right, while a moment later the situation will have reversed. If the present study had made measurements using the standard two-response method (Breese, 1899 ), it is very likely that what are here recorded as occurrences of the mixed state would be found to contain numerous perceptual alternations. There is thus no contradiction between the present finding that exclusive visibility rises as a viewing period progresses and the report by Fox and Herrmann (1967) that the phases of rivalry (using the two-response method in a situation from which the mixed state was essentially absent) do not change systematically in duration over the course of a trial.

Goldstein (1968) studied the course of rivalry by asking subjects to indicate when each eye's target was, and was not, visible in its entirety. Two response keys were used. He reported his data in the form of "number of changes" per 10-sec block, which he found to increase over the first minute or two of viewing. While his data thus support the idea that there are systematic changes in rivalry as a viewing period proceeds, his results cannot be directly compared with those reported here because the tasks of subjects in the two studies were different.

If binocular rivalry consisted of reciprocal inhibition between signals from the two eyes, then the finding of the present study-that suppression becomes more complete as time passes within a trial-could be taken as evidence that the inhibitory mechanism had a high gain. There is, however, formidable evidence against a reciprocal inhibition model of rivalry, particularly the finding (Blake, 1977; Fox \& Rasche, 1969; Levelt, 1965) that changes in the stimulus strength in one eye do not influence the duration of periods for which the other eye's view is suppressed. At the present time, then, there is no ready explanation for the data shown in Figure 3.

\section{Spatial Frequency Action Spectrum of Rivalry}

It is known that two information channels, one responding primarily to changes in stimulation, the other sensitive to spatial patterns and responding in a more sustained way, are at work in human vision (Keesey, 1972; Kulikowski \& Tolhurst, 1973). Recently, Marshak and Sekuler (1979) have suggested that the movement-detecting system is not involved in binocular rivalry. This conclusion was based on their observation that fields of dots, moving in different directions for the two eyes, do not appear to rival.
Marshak and Sekuler's idea is supported by the observations of Breese (1899), who found that when a stationary target was viewed with one eye and a moving target with the other eye, the stationary target appeared and disappeared with regularity, but the moving target was nearly always visible. The lines of the moving grating were not clear when the stationary grating was visible, however. Breese's observations seem made to order for the idea that patterns (whether moving or stationary) engage in rivalry, while the perception of movement itself remains unsuppressed.

On the other hand, there are conditions under which a moving or phase-alternating stimulus can undergo periods of complete suppression (Lehmkuhle \& Fox, 1975), even when the other eye's target is stationary (Blake \& Fox, 1974), and detecting that a test target has begun to move takes longer if the field on which it is superimposed is in a suppressed rather than a dominant phase of binocular rivalry (Fox \& Check, 1968). Moreover, setting a target in motion can increase its ability to dominate a stationary target in the other eye (Fox \& Check, 1968; Springbett, 1961). These observations suggest that signals in the transient channel are not insulated from the rivalry process.

In this context, the finding of the present study that the spatial frequency "action spectrum" of binocular rivalry peaks at about 3 cycles/deg is of interest, for that is the spatial frequency at which the transient system is most sensitive (Kulikowski \& Tolhurst, 1973), a fact confirmed here by control measurements (triangles in Figure 6) on the same subjects who participated in the rivalry experiments. This suggests that the transient system plays a role in rivalry, even for targets whose only retinal image motion is that caused by eye movements. It may be, however, that the agreement between the peak of the rivalry action spectrum and that of the transient system's contrast sensitivity function is fortuitous. The issue is an important one deserving of more experimental study, perhaps involving different types of temporal modulation between which only the transient system can distinguish (Kulikowski \& Tolhurst, 1973).

\section{REFERENCES}

Blake, R. Threshold conditions for binocular rivalry. Journal of Experimental Psychology: Human Perception and Performance, 1977, 3, 251-257.

Blake, R., \& Camisa, J. On the inhibitory nature of binocular rivalry suppression. Journal of Experimental Psychology: Human Perception and Performance, 1979, 5, 315-323.

BLAKE, R., \& Fox, R. Binocular rivalry suppression: Insensitive to spatial frequency and orientation change. Vision Research, $1974,14,687-692$.

Blake, R., \& LEMA, S. A. Inhibitory effect of binocular rivalry suppression is independent of orientation. Vision Research, $1978,18,541-544$.

Blakemore, C., Muncey, J. P. J., \& Ridley, R. M. Perceptual fading of a stabilized cortical image. Nature, 1971, 233, 204-205. 
Blakemore, C., Muncey, J. P. J., \& Ridley, R. M. Stimulus specificity in the human visual system. Vision Research, 1973 , 13, 1915-1931.

Breese, B. B. On inhibition. Psychological Monographs, 1899 3(1, Whole No. 11), 1-65.

Breese, B. B. Binocular rivalry. Psychological Review, 1909, $16,410-415$

Campbell, F. W., \& Green, D. G. Optical and retinal factors affecting visual resolution. Journal of Physiology, 1965, 181, 576-593.

Crovitz, H. F., \& Lockhead, G. R. Possible monocular predictors of binocular rivalry of contours. Perception \& Psychophysics, 1967, 2, 83-85.

Fox, R., \& Check, R. Detection of motion during binocular rivalry suppression. Journal of Experimental Psychology, 1968 78, 388-395

Fox, R., \& Снеск, R. Independence between binocular rivalry suppression duration and magnitude of suppression. Journal of Experimental Psychology, 1972, 93, 283-289.

Fox, R., \& HERMANN, J. Stochastic properties of binocular rivalry alternations. Perception \& Psychophysics, 1967, 2(9), 432-436.

Fox, R., \& Rasche, F. Binocular rivalry and reciprocal inhibition. Perception \& Psychophysics, 1969, 5, 215-217.

Goldstein, A. G. Retinal rivalry and Troxler's effect: A correlation. Perception \& Psychophysics, 1968, 4, $261-263$.

HochBERG, J. Contralateral suppressive fields of binocular combination. Psychonomic Science, 1964, 1, 157-158.

Hollins, M., \& LEUng, E. H. L. The influence of color on binocular rivalry. In J. C. Armington, J. Krauskopf, \& B. R. Wooten (Eds.), Visual psychophysics and physiology. A volume dedicated to Lorrin Riggs. New York: Academic Press, 1978.

Hudnell, H. K., \& Hollins, M. Fatigue of the suppression mechanism following prolonged binocular rivalry. Investigative
Ophthalmology and Visual Science, 1979, Supplement, p. 173. KEESEY, U. T. Flicker and pattern detection: A comparison of thresholds. Journal of the Optical Society of America, 1972, 62, 446-448.

Kulikowski, J. J., \& Tolnurst, D. J. Psychophysical evidence for sustained and transient detectors in human vision. Journal of Physiology, 1973, 232, 149-162.

Leнмкuhle, S. W., \& Fox, R. Effect of binocular rivalry suppression on the motion aftereffect. Vision Research, 1975, 15, 855-859.

LeVelt, W. J. M. On binocular rivalry. Soesterberg, The Netherlands: Institute for Perception RVO-TNO, 1965.

Marshak, W., \& Sekuler, R. Mutual repulsion between moving visual targets. Science, 1979, 205, 1399-1401.

OGLE, K. N., \& W AKEFIELD, J. M. Stereoscopic depth and binocular rivalry. Vision Research, 1967, 7, 89-98.

Panum, P. L. Physiologische Untersuchungen über das Sehen mit zwei Augen. Kiel, Germany: Schwerssche Buchhandlung, 1858.

SpringBetT, B. M. Some stereoscopic phenomena and their implications. British Journal of Psychology, 1961, 52, 105-109.

WADE, N. J. The effect of orientation in binocular contour rivalry of real images and afterimages. Perception \& Psychophysics, $1974,15,227-232$.

WADE, N. J. Monocular and binocular rivalry between contours. Perception, 1975, 4, 85-95.

Wales, R., \& Fox, R. Incremental detection thresholds during binocular rivalry suppression. Perception \& Psychophysics, $1970,8,90-94$.

(Received for publication May 30, 1979; revision accepted March 5, 1980.) 